ESAIM: PROCEEDINGS, August 2010, Vol. 30, p. 34-43

D. Bresch, V. Calvez, E. Grenier, P. Vigneaux \& J.-F. Gerbeau, Editors

\title{
OPTIMAL PLACEMENT OF ELECTRODES IN AN ELECTROPORATION PROCESS
}

\author{
Nicolae Cîndea ${ }^{1}$, Benoît Fabrèges ${ }^{2}$, Frédéric de Gournay $^{3}$ and Clair \\ POIGNARD $^{4}$
}

\begin{abstract}
Electroporation consists in increasing the permeability of a tissue by applying high voltage pulses. In this paper we discuss the question of optimal placement and optimal loading of electrodes such that electroporation holds only in a given open set of the domain. The electroporated set of the domain is where the norm of the electric field is above a given threshold value. We use a standard gradient algorithm to optimize the loading of the electrodes and shape sensitivity analysis and a gradient algorithm in order to move the electrodes. We also discuss the choice of objective functions to be chosen in the gradient algorithm.

Résumé. L'électroporation est un procédé qui consiste à augmenter la perméabilité d'un tissu en le soumettant à des pulses électriques de grande amplitude. L'objet de ce papier est d' étudier l'optimisation du placement des électrodes afin d'électroporer une région d'un domaine d'étude. La partie électroporée est celle où la norme du champ électrique dépasse une valeur seuil. Nous utilisons une méthode de gradient standard afin d'optimiser la charge des électrodes. L'optimisation du placement des électrodes est effectuée à l'aide d'une dérivation de domaine et d'un algorithme de gradient. Nous concluons par des simulations qui illustrent l'efficacité de la méthode proposée.
\end{abstract}

\section{INTRODUCTION}

Electrochemotherapy is an effective non-thermal cancer treatment for solid metastases that consists in applying simultaneously intra-tumoral drug delivery and high-voltage short pulses to the tumor [5]. The pulses increase locally the permeability of the cells - such a phenomenon is called electroporation or electropermeabilization $[3,10,13,16]$ - allowing the drugs to enter into tumor. When appropriately used, the electrochemotherapy is very efficient: since drugs enter the cancer cell cytoplasm, the treatment efficacy is multiplied by thousand compared with usual chemotherapy [5]. Nevertheless clinical applications require a precise control of both electric field distribution and amplitude in the tissues. Actually the electric field has to be sufficiently high in the whole tumor without causing damages in the surrounding healthy tissues.

\footnotetext{
${ }^{1}$ Institut Elie Cartan, Nancy Université / CNRS / INRIA, BP 70239, 54506 Vandoeuvre-lès-Nancy, France. mailto :Nicolae.Cindea@iecn.u-nancy.fr

${ }^{2}$ Université Paris Sud XI, Orsay, France.

mailto :benoit.fabreges@math.u-psud.fr

${ }^{3}$ LMV, Université Versailles-Saint Quentin, CNRS, Versailles, France.

mailto :gournay@math.uvsq.fr

${ }^{4}$ EPI MC2, INRIA Bordeaux-Sud Ouest \& Institut de Mathématiques de Bordeaux, Talence, France. mailto :clair.poignard@inria.fr
}

(C) EDP Sciences, SMAI 2010 
From the bioelectrical engineering point of view, a tumor is a lossy material with a conductivity larger than the conductivity of the healthy tissues $[9,12,14]$. In this paper, we suppose that the tumor is entirely surrounded by a homogeneous healthy tissue. The electrodes are considered as perfectly conducting materials since their conductivity is much larger than the biological tissues conductivity. In order to achieve efficiently the electroporation of the tumor, the electric field in the tumor must reach the reversible threshold value $E_{\text {rev }}$ without overcoming the irreversible threshold $E_{\text {irr }}$ in the surrounding tissue, in order to prevent damages such as burning, that can dramatically slow down the healing. In addition, since we do not want drugs to enter the healthy tissue, we ideally would impose that the electric field is under $E_{\mathrm{rev}}$ in the healthy tissue. However, such a condition is difficult to enforce because the boundary of a tumor cannot be determined precisely-the probability of finding tumor cells in a small neighborhood of the boundary of a solid is not negligible- and therefore for safety reason the surgeon used to electroporate the region of the healthy tissue that is very close to the tumor.

We aim at providing an efficient algorithm, that optimizes at the same time the placement of the electrodes and the voltage imposed on each electrode in order to electroporate efficiently a tissue with a tumor. This paper can be seen as a first step in the optimization of the electrochemotherapy treatment. Particularly, the problem we address here is much simpler than the "reality". Actually, we only consider here time-harmonic electric field, and the conductivities of the tissue are supposed constant. Moreover, the thermal effects due to electromagnetic fields are omitted. In addition the electrochemotherapy treatments consist in applying several electric micropulses-a micropulse is a pulse with duration 1 microsecond- and only two electrodes can be switched on for each delivery, while we consider here only one pulse and all the electrodes can be switched on at the same time. However, we are confident that the present results provide a new insight in the optimization placement of the electrodes. Actually, several papers have been devoted to tackle this problem using bioelectrical engineering approach $[11,12]$. In these previous papers, the electrodes placement is a priori chosen, and numerical simulations are performed for the different chosen configurations, providing non accurate results from the optimization point of view. In this paper, the optimization is performed using a two-step algorithm based on the steepest descent method and the derivation of the objective function is obtained by a shape sensitivity analysis [1].

In the next section, we set the equations that are to be solved, and we make precise the choice of the objective function to be minimized. Section 3 is devoted to the optimization of the voltage, that is to be imposed on each electrode, while in Section 4 we optimize the electrode placement. Numerical results are given in Section 5 . We conclude by proposing future research directions, that we would like to follow.

\section{Setting the Problem}

Let $\Omega$ be a simply connected open set in $\mathbb{R}^{d}, d=2$ or 3 , with a $C^{1}$-boundary $\partial \Omega$. The conductivity map of $\Omega$ is denoted by $\sigma \in L^{\infty}(\Omega)$. For $N \geq 2$, let $\left(V_{i}\right)_{i=1, \cdots, N}$ be $N$ subdomains of $\Omega$ and denote by $\left(a_{i}\right)_{i=1, \cdots, N} N$ reals numbers. We consider the steady-state potential $u$, which is the unique solution to:

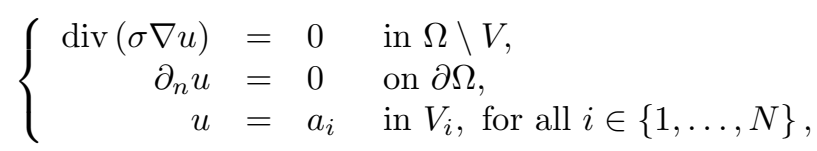

where $V=\cup_{i} V_{i}$.

Denote by $E_{\text {rev }}$ and $E_{\text {irr }}$ the reversible and the irreversible electroporation threshold values, respectively. The tumor is modelled by a target open set $\omega \subset \Omega$. The goal of this paper is to find, for given $N$, the optimized parameters $a_{i}$ and electrodes position $V_{i}$ such that

$$
\left\{\begin{aligned}
|\nabla u|<E_{\text {rev }} & \text { in } \Omega \backslash \omega \\
E_{\text {rev }} \leq|\nabla u|<E_{\text {irr }} & \text { in } \omega .
\end{aligned}\right.
$$


In clinical applications of electrochemotherapy, only few shapes of electrodes are at the surgeon's disposal [9, 15]. Therefore, we do not study in the present paper the optimal design of electrodes. We consider only the case of needle shaped electrodes, which are very commun in clinical applications and easiest to handle from a numerical point of view.

The motion of each electrode $V_{i}$ is described by a rotation $R_{i}$ around the mass center of the electrode and a translation $e_{i}$. Moreover we add the constraint of non-overlapping. In order to achieve objective (2), we need the so-called objective function $\mathcal{J}$ defined by

$$
\mathcal{J}=\int_{\Omega \backslash V} \chi(x) g(|\nabla u(x)|-\Phi(x)) \mathrm{d} x
$$

where $g$ is a function such that $x=0$ is the unique minimizer (typically $g(x)=x^{2}$ ), $\Phi$ is a target function, for instance:

$$
\begin{cases}\Phi=0, & \text { in } \Omega \backslash \omega \\ \Phi=\left(E_{\text {rev }}+E_{\text {irr }}\right) / 2, & \text { in } \omega,\end{cases}
$$

and $\chi$ is a weight function that discriminates different regions of interest. We aim to minimize $\mathcal{J}$ on the set of the rotations $R_{i}$, the translations $e_{i}$ and the voltage $a_{i}$. This functional $\mathcal{J}$ tends to set $|\nabla u|$ close to $\Phi$, hence partially achieving the sought goal. In order to reach the minimum $\min _{a_{i}, R_{i}, e_{i}} \mathcal{J}$, we use the following twosteps optimization algorithm : for given voltages $\left(a_{i}^{t}\right)_{i=1, \cdots, N}$ at the step $t$, we optimize the electrode position $\left.\left(V_{i}^{t}\right)\right)_{i=1, \cdots, N}$. Then, given the positions $\left(V_{i}^{t}\right)_{i=1, \cdots, N}$, we optimize the voltages $\left(a_{i}^{t+1}\right)_{i=1, \cdots, N}$ at the following step $t+1$.

\section{Voltage optimization}

For given electrodes positions $V_{i}$, the optimization problem is to minimize the objective function with respect to the voltages $a_{i}$. For each $i$ such that $1 \leq i \leq N$, we compute $u_{i}$, the solution to

$$
\left\{\begin{array}{rll}
\operatorname{div}\left(\sigma \nabla u_{i}\right) & =0 & \text { in } \Omega \backslash V \\
\partial_{n} u_{i} & =0 & \text { on } \partial \Omega, \\
u_{i} & =1 & \text { in } V_{i}, \quad u_{i}=0 \text { in } V_{j}, \text { for } j \neq i .
\end{array}\right.
$$

According to the superposition principle $u=\sum_{i} a_{i} u_{i}$, hence the voltage optimization is reduced to

$$
\min _{a_{i}} \mathcal{J}(a) \quad \text { with } \mathcal{J}(a)=\int_{\Omega \backslash V} \chi(x) g\left(\left|\sum_{i} a_{i} \nabla u_{i}(x)\right|-\Phi(x)\right) \mathrm{d} x .
$$

We stress that the above problem is an optimization problem with $N$ unknowns, which is a costless problem compared to the computation of the $\left(u_{i}\right)_{i=1, \cdots, N}$. Observe that if every $a_{i}$ is shifted with the same constant, then $\nabla u$ does not change, hence the objective function remains constant. We thus set $a_{1}=0$ and optimize only with respect to the $N-1$ variables $\left(a_{i}\right)_{i=2, \cdots, N}$. We use a gradient method with a 10 steps line search algorithm in order to compute the minimum.

\section{Optimal Electrode Placement}

We perform the optimization of the electrodes position using the shape sensitivity analysis [7]. For any $\theta$ belonging to $W^{1, \infty}(\Omega)$, we denote by $T$ the application $T=I d+\theta$. The function $u_{\theta}$ is then the solution to problem (1), replacing $\Omega$ and $V_{i}$ respectively by $T(\Omega)$ and $T\left(V_{i}\right)$. Here, $\theta$ is the vector field that induces the diffeomorphism $T$ which transports the electrodes. We assume that the tumor is located far from the boundary of $\Omega$. Since we do not want to change the shape of $\Omega$, we impose $T(\Omega)=\Omega$ and more precisely we impose $\theta=0$ in a neighborhood of $\partial \Omega$. 
Since the space of vector fields in $W^{1, \infty}(\Omega)$ is a Banach space, there is a notion of derivability with respect to $\theta$. Showing the derivability of $\mathcal{J}$ and computing it is quite a long process, there exists an heuristic shortcut known as "the Lagrangian method of Céa" [2]. We emphasize that this shortcut is not rigorous from a mathematical point of view since this method supposes that the derivability of $\mathcal{J}$ with respect to vector fields has already been proven. Nevertheless, we present here this method for the sake of simplicity, and since it provides an explanation of the derivation process, which is rigorously proved in [7].

Let $a$ be a function equal to $a_{i}$ in a neighborhood of each $V_{i}$ and introduce the Lagrangian defined for any $\left(v, q, q_{d}\right) \in\left(H^{1}\left(\mathbb{R}^{d}\right)\right)^{3}$ by

$$
\mathcal{L}\left(v, q, q_{d}\right)=\int_{\Omega \backslash V} \chi g(|\nabla v|-\Phi)-\int_{\Omega \backslash V} \sigma \nabla v \nabla q+\int_{\partial V}\left(\sigma \partial_{n} v\right) q+\int_{\partial V}(v-a) q_{d},
$$

where $n$ denotes the normal to $\partial V$ outwardly directed from $\Omega \backslash V$ (hence $n$ is directed into $V$ ). Then minimizing $\mathcal{L}$ on both $q$ and $q_{d}$ yields $v=u$ and hence

$$
\mathcal{J}=\max _{v \in H^{1}\left(\mathbb{R}^{d}\right)} \min _{q, q_{d} \in H^{1}\left(\mathbb{R}^{d}\right)} \mathcal{L}\left(v, q, q_{d}\right) .
$$

The saddle point of the functional $\mathcal{L}$ is $\left(u, p, p_{d}\right)$, where $p$ is the so-called "adjoint state" defined by

$$
\left\{\begin{aligned}
\operatorname{div}(\sigma \nabla p) & =\operatorname{div}\left(\chi g^{\prime}(|\nabla u|-\Phi) \frac{\nabla u}{|\nabla u|}\right) & & \text { in } \Omega \backslash V, \\
\sigma \partial_{n} p & =\chi g^{\prime}(|\nabla u|-\Phi) \frac{\partial_{n} u}{|\nabla u|} & & \text { on } \partial \Omega, \\
p & =0 & & \text { in } V,
\end{aligned}\right.
$$

and the Lagrange multiplier $p_{d}$ satisfies

$$
p_{d}=\sigma \partial_{n} p-\chi g^{\prime}(|\nabla u|-\Phi) \frac{\partial_{n} u}{|\nabla u|} \text { on } \partial V .
$$

Deriving $\mathcal{J}$ with respect to the domain amounts to deriving $\mathcal{L}$ with respect to the domain and to taking the derivative at the saddle point since, and it is the key point of this method, the domains on which the minimizers and maximizers are sought are independent of $\theta$.

In order to compute this derivative, suppose that $f$ is a given function independent on $\theta$. According to [1], the derivation of

$$
\int_{T(\Omega)} f \text { and } \int_{T(\partial \Omega)} f
$$

is computed using a change of variables and leads respectively to:

$$
\int_{\partial \Omega}(\theta \cdot n) f \text { and } \int_{\partial \Omega}(\theta \cdot n)\left(H f+\partial_{n} f\right) .
$$

Therefore, since $\theta$ vanishes on $\partial \Omega$, we infer that

$$
\begin{aligned}
\mathcal{J}^{\prime} & =\int_{\partial V}(\theta \cdot n)(\chi g(|\nabla u|-\Phi)-\sigma \nabla u \nabla p) \\
& +\int_{\partial V}(\theta \cdot n) H\left(\sigma \partial_{n} u p+(u-a) p_{d}\right)+\partial_{n}\left(\sigma \partial_{n} u p+(u-a) p_{d}\right),
\end{aligned}
$$

where $n$ is the (inner) normal to $\partial V$. Using $u=a, p=0$ on $\partial V$, and equation (6), the expression of $\mathcal{J}^{\prime}$ simplifies into

$$
\mathcal{J}^{\prime}=\int_{\partial V}(\theta \cdot n) j,
$$


where

$$
j(x)=\chi g(|\nabla u|-\Phi)-|\nabla u| \chi g^{\prime}(|\nabla u|-\Phi)+\sigma \partial_{n} u \partial_{n} p .
$$

On each electrode, $\theta$ is restricted to the velocity field of a rigid motion. Moreover, in two dimensions, the electrodes are supposed to be radially symmetric and then $\theta$ can be restricted to a translation. Hence there exists vectors $e_{i}$ and $R_{i}$ such that, near the $i^{t h}$-electrode, we have :

$$
\theta(x)=\left(x-x_{i}\right) \wedge R_{i}+e_{i} \text { for the } 3 d \text { case, } \quad \text { and } \quad \theta(x)=e_{i} \text { for the } 2 d \text { case }
$$

where $x_{i}$ is the coordinate of the center of mass of electrode $i$. Plugging this additional hypothesis in (8), we infer that

$$
\mathcal{J}^{\prime}=\sum_{i} R_{i} \cdot \alpha_{i}+e_{i} \cdot \beta_{i}
$$

where the parameters $\alpha_{i}$ and $\beta_{i}$ are given by :

$$
\alpha_{i}=\int_{\partial V_{i}} n \wedge\left(x-x_{i}\right) j \quad \text { and } \quad \beta_{i}=\int_{\partial V_{i}} n j .
$$

At each iteration of the gradient algorithm, we choose $R_{i}=-\alpha_{i}$ (in $3 d$ ) and $e_{i}=-\beta_{i}$ in order to minimize $\mathcal{J}$.

\section{Numerical Simulations}

The numerical simulations have been computed in $\mathrm{C}++$ using the mesh generator Gmsh [4] and the finite element library GetFEM++ [8].

For numerical purposes, the electrodes are modelled via a parametrized level set. In $2 \mathrm{~d}$, remeshing after each movement of the electrodes is possible and not very costly.

To avoid remeshing each time the electrodes are moved, we use a penalty method for solving (1) and (5). We aim to solve numerically (1), using finite elements method. For this purpose, we consider the variational formulation of (1) :

$$
\text { Find } u \in \mathcal{K} \text { such that } \int_{\Omega} \sigma \nabla u \cdot \nabla \varphi=0 \quad(\varphi \in \mathcal{K}) \text {, }
$$

where $\mathcal{K}=\left\{v \in H^{1}(\Omega) \mid v=a_{i}\right.$ in $\left.V_{i}\right\}$. The idea of the penalty method is to replace the variational problem (9), which is formulated in $\mathcal{K}$, by another variational problem in $H^{1}(\Omega)$. Therefore, for a given $\varepsilon>0$, we consider the following problem :

$$
\text { Find } u \in H^{1}(\Omega) \text { such that } \quad \int_{\Omega} \sigma \nabla u \cdot \nabla \varphi+\frac{1}{\varepsilon} \sum_{i=1}^{N} \int_{V_{i}}\left(u-a_{i}\right) \varphi=0 \quad\left(\varphi \in H^{1}(\Omega)\right) .
$$

It is known that the solution of (10) is obtained as the minimum of the following functional:

$$
J(u)=\frac{1}{2} \int_{\Omega}|\sigma||\nabla u|^{2}+\frac{1}{2 \varepsilon} \sum_{i=1}^{N} \int_{V_{i}}\left|u-a_{i}\right|^{2} .
$$

Therefore, if $\varepsilon>0$ is small enough, the solution $u$ of (10) will be "close" to the solution of (9) in $\Omega \backslash V$ and "close" to $a_{i}$ in $V_{i}$ for every $i \in\{1, \ldots, N\}$. A precise formulation of these results and a proof can be found in [6]. A similar penalized variational problem is solved for the adjoint problem (5).

Observe that the electrodes are relatively small compared with the tumor. Therefore, since the formula for the computation of the shape derivative is expressed on the boundary of the electrodes, the mesh has to be refined near each $V_{i}$ in order to compute accurately the shape sensitivity analysis.

We choose to refine the mesh on the boundary of each electrode. Recall that the electrodes are completely described by their positions and sizes. Therefore, we define a level-set corresponding to each electrode by a 
function being 0 on the boundary of electrode, positive outside the electrode and negative in the interior of the electrode. For example, the level-set correspondig to a circular electrode $V_{i}$ of center $C_{i}\left(x_{i}, y_{i}\right)$ and radius $r_{i}$ is the function $L_{i}$ given by

$$
L_{i}(x, y)=\left(x-x_{i}\right)^{2}+\left(y-y_{i}\right)^{2}-r_{i}^{2}, \quad \text { for every }(x, y) \in \Omega .
$$

A similar level-set function is considered in three-dimensional case.

With these notations, to refine the mesh on the boundary of the $i$-th electrode we compute the intersection of each mesh element (triangles in $2 \mathrm{~d}$ and tetrahedra in $3 \mathrm{~d}$ ) with the set described by $L_{i}=0$. If this intersection is non-empty, we cut the element along a simplex included in the intersection (a line segment in $2 \mathrm{~d}$ and a triangle in 3d). Moreover, if necessary in the purpose of obtaining the same kind of finite elements as before remeshing, we realize some more cuts. In this way we obtain a mesh on the surface of the electrode and we can approximate the value of $\mathcal{J}^{\prime}$.

\subsection{Numerical results}

The $2 d$ tests are performed in the squared domain $\Omega=[0,1] \times[0,1]$ meshed with $11 \mathrm{~K}$ triangles. The electric potentials and the adjoint problem are solved using $P 1$ finite elements and an iterative solver with a $P 0$ conductivity equals to 0.4 in the healthy tissue, and 1 in the tumor.

For the first serie of tests, the tumor is an ellipse with center $[0.5,0.5]$ and semi-axis of sizes 0.2 and 0.3 , in the directions $x$ and $y$, respectively. The electrodes are disks of radius 0.02 . The ponderation of the zone of interest $\chi$ is equal to 10 in the tumor and 1 in the healthy tissue, meaning that we are 10 times more interested in satisfying the conditions inside the tumor than inside the healthy tissue. Several tests are performed with an increasing number of electrodes. Results are given in Table 1 and the optimal potential and electrodes placement is shown in Figure 1, whereas the convergence history is shown in Figure 2.

\begin{tabular}{|l|c|r|}
\hline $\begin{array}{l}\text { Name } \\
\text { (Number of elec.) }\end{array}$ & $\begin{array}{c}\text { Initial position of the } \\
\text { electrodes }\end{array}$ & Final energy \\
\hline \hline 2 & $(0.2 ; 0.2)$ and $(0.8,0.8)$ & 3.00259 \\
3 & same as $2+(0.8 ; 0.2)$ & 2.96855 \\
4 & same as $3+(0.2 ; 0.8)$ & 2.45406 \\
6 & same as $4+(0.1,0.5)+(0.9,0.5)$ & 2.22236 \\
$6 \mathrm{~b}$ & same as $4+(0.5,0.1)+(0.5,0.9)$ & 2.85978 \\
\hline
\end{tabular}

TABLE 1. The initial position of the electrodes and the final value of the objective function $\mathcal{J}$ for $2 d$ tests.

This $2 d$ algorithm shows the right behavior. Generally, the more electrodes we use, the more degrees of freedom has the algorithm to optimize their positions. Tests 2 and 3 yield the same result, the added third electrode being useless and it is always "switch-off" during the optimization process, which means that it is always set to a potential that is the potential at the location of the electrode when it is removed.

Test 4 exhibits a two-step behavior. During iterations 1 to 5 , it only optimizes the position of the two electrodes that are in location $(0.2,0.2)$ and $(0.8,0.8)$, switching off the other two electrodes. This can be seen in Figure 1 where the curve for 4 electrodes is similar to the one for 2 electrodes during the beginning of the optimization process. After having optimized the position of these two electrodes, it is concerned by two remaining ones, moving them slowly close to the tumor during iterations 10 to 22 .

Test 6 stops in a local optimum. Indeed, the final configuration is very similar to the previous one but the energy is a little higher. This phenomenon is due, probably, to the fact that the algorithm has more electrodes to move together, which is harder to optimize near a minimum. Moreover test $6 b$ shows that there exists a better configuration that is completely different from the one of test 6 . Remark that the particular position 


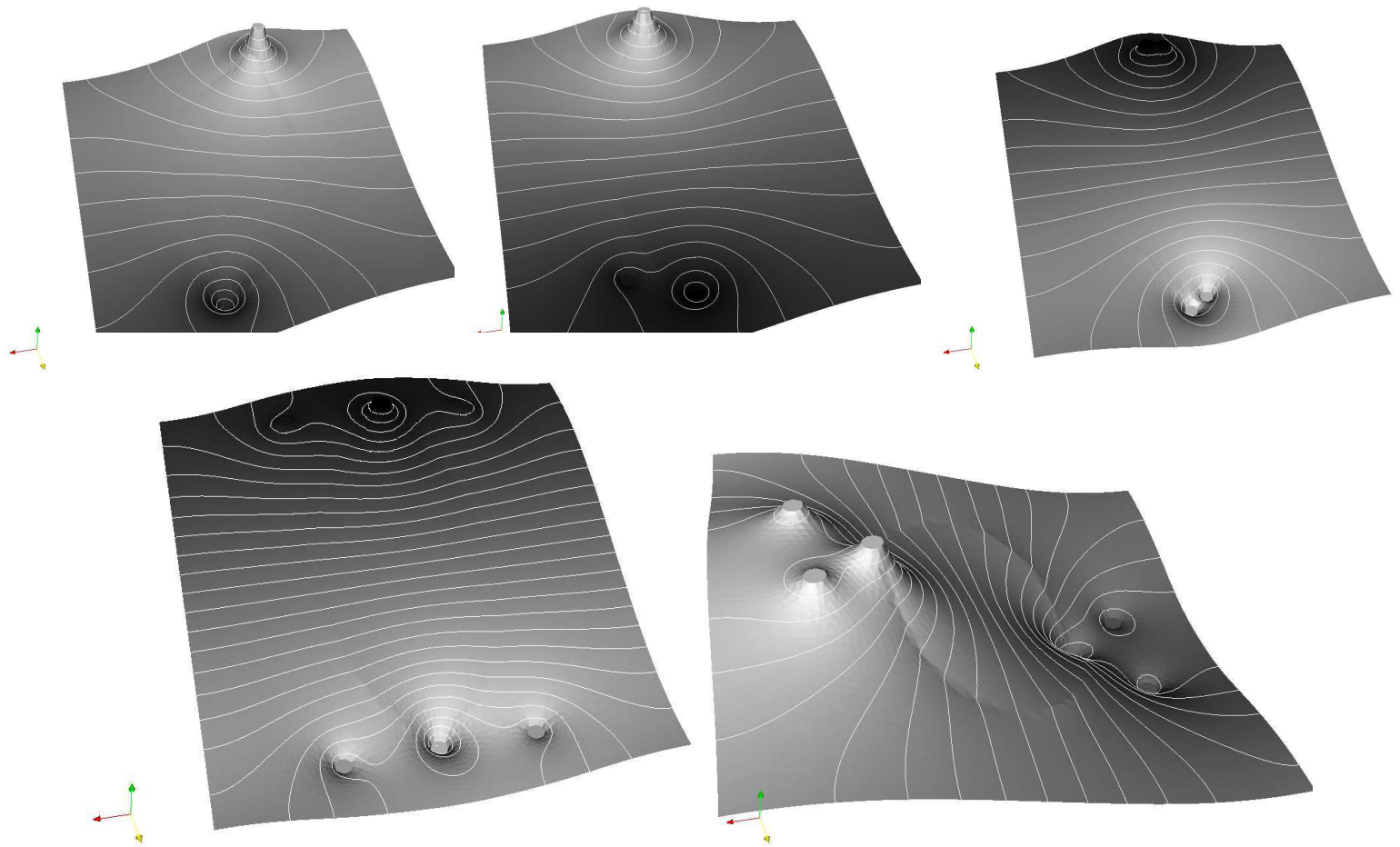

FIgURE 1. The electric potential for the optimal electrodes position in the $2 \mathrm{~d}$ test. From left to right and from top to bottom, the number of electrodes is respectively $2,3,4,6,6 \mathrm{~b}$ of Table 1

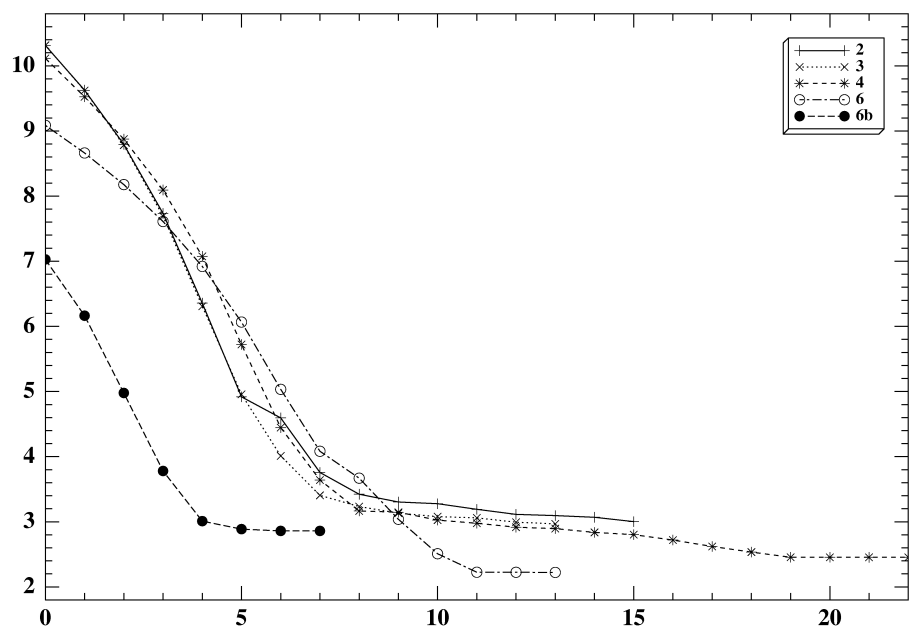

FiguRE 2. Convergence history of the objective function for the different electrodes in $2 \mathrm{~d}$ case. Abscissa refers to the number of iterations.

of electrodes in $6 b$ is very close to one of the configurations used in medical practice : two plates disposed symmetrically on the both sides of the tumor. 
The $3 \mathrm{~d}$ tests are made on the cube $\Omega=[0,1]^{3}$ meshed with aproximatively $35 \mathrm{~K}$ tetrahedra. As for $2 \mathrm{~d}$ tests, the electric potentials and the adjoint problem are solved using $P_{1}$ finite elements and the conductivity $\sigma$, represented with $P_{0}$ finite elements, is equal to 0.4 in the healthy tissue and 1 in the tumor.

We consider a spherical tumor centered in $(0.5,0.5,0.5)$ and with the diameter equals to 0.4 . The electrodes are circular cylinders of diameter 0.1. The set of all possible electrodes configurations is subject to some restrictions : first of all electrodes have vertical position and the intersection with the plane of equation $z=1$ is non-empty. Therefore, the three coordinates of the center of the lower base of the electrode describe completely its position.

The ponderation of the zone of interest $\chi$ is equal to 10 in the tumor and 1 in the healthy tissue, meaning that we are 10 times more interested in satisfying the conditions inside the tumor than inside the healthy tissue. Several tests are performed with 2, 4 or 6 electrodes. Results are given in Table 2 and the optimal potential and electrodes placement is shown in Figure 3, whereas the convergence history is shown in Figure 4.

\begin{tabular}{|l|c|r|}
\hline $\begin{array}{l}\text { Name } \\
\text { (Number of elec.) }\end{array}$ & $\begin{array}{c}\text { Initial position of the } \\
\text { electrodes }\end{array}$ & Final energy \\
\hline \hline 2 & $(0.5,0.2,0.7)$ and $(0.5,0.8,0.7)$ & 1.03087 \\
\hline 4 & $\begin{array}{l}(0.5,0.2,0.7),(0.8,0.5,0.7) \\
(0.5,0.8,0.7),(0.2,0.5,0.7)\end{array}$ & 0.807027 \\
\hline 6 & $\begin{array}{l}\{0.2,0.5,0.8\} \times\{0.2\} \times\{0.7\} \\
\{0.2,0.5,0.8\} \times\{0.8\} \times\{0.7\}\end{array}$ & 0.803599 \\
\hline
\end{tabular}

TABLE 2. The initial position of the electrodes and the final value of the objective function (sometimes called energy) in the $3 d$ tests. The first column displays the number of electrodes.

The results obtained in the $3 \mathrm{~d}$ tests are similar to the results obtained in $2 \mathrm{~d}$. All the optimal configurations displayed in Figure 3 shows that, even for more than two electrodes, the energy is minimized by a pair of electrodes placed symmetrically around the tumor. This can be explained by the important size of the electrodes compared with the tumor.

\section{Conclusion}

In this paper we have proposed a numerical method to optimize the position and the voltage of the electrodes in order to electroporate the biggest area of a tumor and the smallest area of the healthy tissue. This method is based on a minimization of an objective functional using the steepest descent method. Although the concept is not new, its application to the electrochemotherapy have not been performed yet and this paper provides a first step in this direction. Actually, we are confident that such an approach can provide efficient and precise tools to tackle the problems of the optimal delivery of pulses in a tumor. As described above, depending on the tumor shape and on its localization, the electrochemotherapy is performed in several steps, using different shapes of electrodes with different numbers of electrodes and pulses. All these considerations have not been tackled in this paper. In particular, we do not know either whether the application of fews high voltage pulses is more efficient than more pulses with lower amplitude or whether the duration of the pulses (100 $\mu \mathrm{s}, 200 \mu \mathrm{s})$ is preponderant. Note also, the living tissues are not "usual" electrical materials, in particular their electrical parameters dependon the electric field and are also time-dependent. Hence the precise models of biological tissues are non linear, whereas we only consider linear P.D.E. Moreover, the conductivity of the electroporated tissue is different from the conductivity of the non-electroporated tissue and, since the tissue recovers its initial conductivity after several minutes, the time dependance of the electrical parameters has to be taken into account. In addition, thermal effects due to the electric field have been omitted and they should be taken into account for medical applications. Therefore, the optimization of the electrochemotherapy is a wide challenge that raises many problems for the applied mathematicians, and in this paper we have presented preliminary results in order to take it up. 

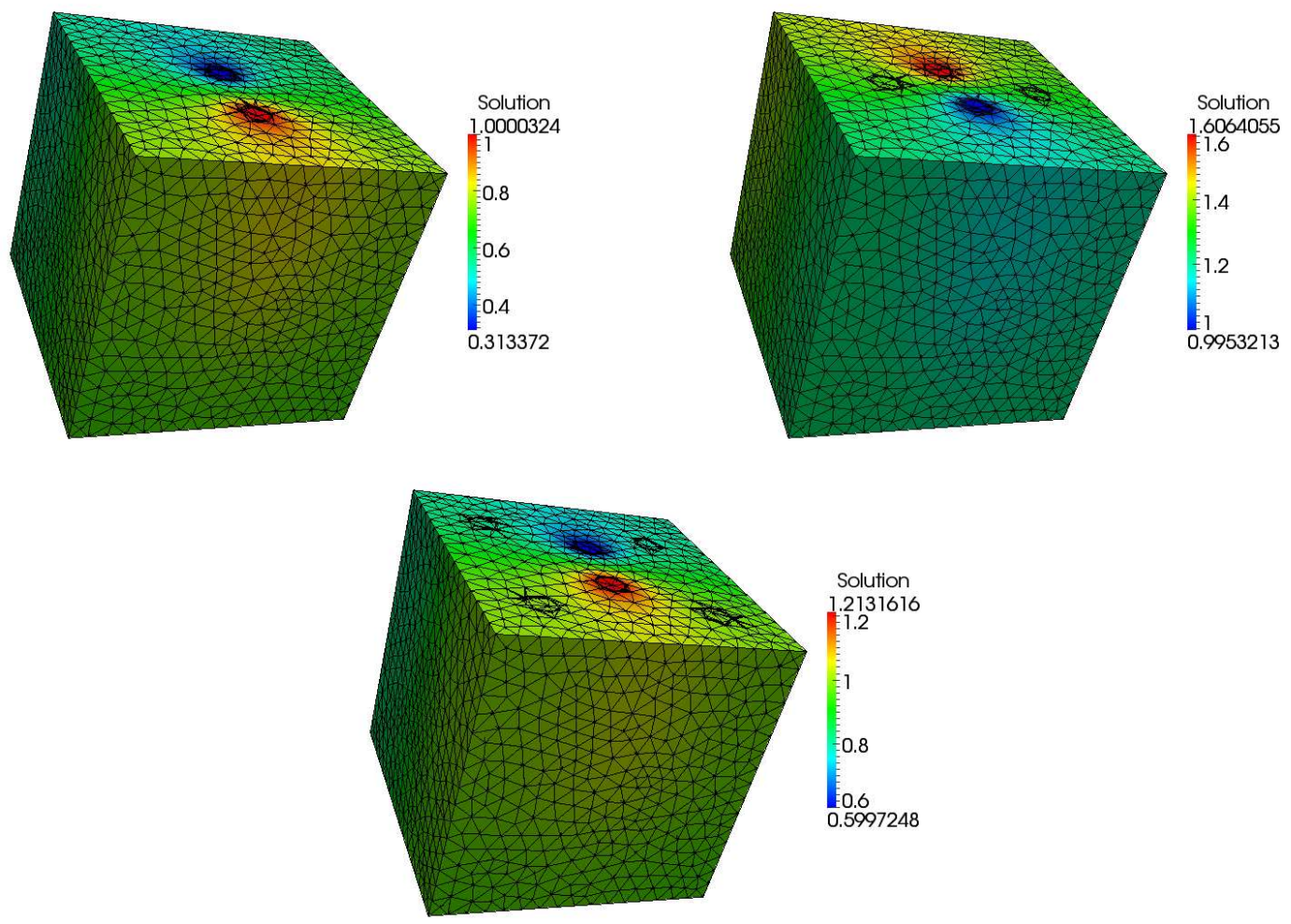

FIGURE 3. The electric potential for the optimal placement of electrodes in 3d, with tests (from left to tight and top to bottom) number 2,4 and 6 of Table 2

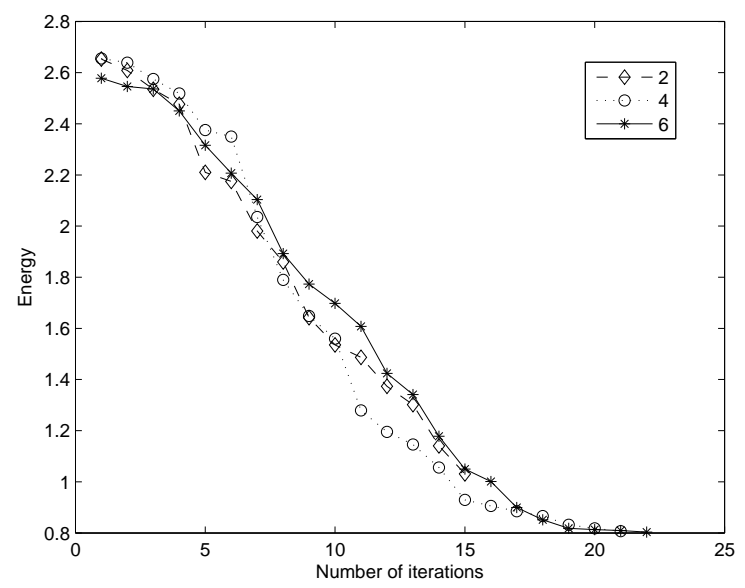

FIGURE 4. Convergence history of the objective function for the different numbers of electrodes in $3 \mathrm{~d}$ case. Abscissa refers to the iteration number.

\section{ACKNOWLEDGEMENT}

This research was partially supported by the ARC C3MB. 


\section{REFERENCES}

[1] Grégoire Allaire. Conception optimale de structures, volume 58 of Mathématiques \& Applications (Berlin) [Mathematics \&3 Applications]. Springer-Verlag, Berlin, 2007. With the collaboration of Marc Schoenauer (INRIA) in the writing of Chapter 8.

[2] Jean Céa. Conception optimale ou identification de formes: calcul rapide de la dérivée directionnelle de la fonction coût. RAIRO Modél. Math. Anal. Numér., 20(3):371-402, 1986.

[3] B. Gabriel and J. Teissié. Time courses of mammalian cell electropermeabilization observed by millisecond imaging of membrane property changes during the pulse. Biophys. J., 76:2158-2165 (electronic), 1999.

[4] C. Geuzaine and J. F. Remacle. Gmsh mesh generator. http://geuz.org/gmsh.

[5] M. Marty, G. Sersa, and J.-R. Garbay et al. Electrochemotherapy - an easy, highly effective and safe treatment of cutaneous and subcutaneous metastases: Results of esope (european standard operating procedures of electrochemotherapy) study. E.J.C Supplements, 4:3-13, 2006.

[6] Bertrand Maury. Numerical analysis of a finite element/volume penalty method. SIAM J. Numer. Anal., 47(2):1126-1148, 2009.

[7] F. Murat and S. Simon. études de problèmes d'optimal design. Lecture Notes in Computer Science 41, Springer Verlag, pages 54-62, 1976.

[8] Y. Renard and J. Pommier. Getfem finite element library. http://home.gna.org/getfem.

[9] G. Serša, D. Miklavčič, M. Cemazar, Z. Rudolf, G. Pucihar, and M. Snoj. Electrochemotherapy in treatment of tumours. EJSO. The Journal of Cancer Surgery, 34:232-240, 2008.

[10] S.I. Sukharev, V.A Klenchin, S.M. Serov, Chernomordik L.V., and Chizmadzhev Y.A. Electroporation and electrophoretic DNA transfer into cells: The effect of DNA interaction with electropores. Biophys J., 63:1320-1327, 1992.

[11] S. Čorovič, J. Bester, and D. Miklavčič. An e-learning application on electrochemotherapy. BioMedical Engineering OnLine, 8, 2009.

[12] S. Črovič, M. Pavlin, and D. Miklavčič. Analytical and numerical quantification and comparison of the local electric field in the tissue for different electrode configurations. BioMedical Engineering OnLine, 6, 2007.

[13] M.C. Vernhes, P.A. Cabanes, and J. Teissié. Chinese hamster ovary cells sensitivity to localized electrical stresses. Bioelectrochem. Bioenerg., 48:17-25, 1999.

[14] D. Šel, D. Cukjati, D. Batiuskaite, T. Slivnik, L.M. Mir, and D. Miklavčič. Sequential finite element model of tissue electropermeabilization. IEEE Trans. Bio. Eng., 52(5):816-827, 2005.

[15] D. Šel, S. Mazeres, and D. Miklavčič. Finite-element modeling of needle electrodes in tissue from the perspective of frequent model computation. IEEE Trans. on BioMed. Eng., 50(11):1221-1232, 2003.

[16] J.C Weaver. Electroporation of cells and tissues. IEEE Trans. on Plasma Sci., 28, 2000. 